\title{
Existence of Solutions and Nonnegative Solutions for Prescribed Variable Exponent Mean Curvature Impulsive System Initialized Boundary Value Problems
}

\author{
Guizhen Zhi, ${ }^{1}$ Liang Zhao, ${ }^{2}$ Guangxia Chen, ${ }^{3}$ \\ Xiaopin Liu, ${ }^{1}$ and Qihu Zhang ${ }^{1,4}$ \\ ${ }^{1}$ Department of Mathematics and Information Science, Zhengzhou University of Light Industry, \\ Zhengzhou, Henan 450002, China \\ ${ }^{2}$ Academic Administration, Henan Institute of Science and Technology, Xinxiang, Henan 453003, China \\ ${ }^{3}$ School of Mathematics and Informatics, Henan Polytechnic University, Jiaozuo, Henan 454003, China \\ ${ }^{4}$ School of Mathematics and Statistics, Huazhong Normal University, Wuhan, Hubei 430079, China
}

Correspondence should be addressed to Qihu Zhang, zhangqh1999@yahoo.com.cn

Received 10 May 2009; Accepted 23 January 2010

Academic Editor: Ondřej Dosly

Copyright (C 2010 Guizhen Zhi et al. This is an open access article distributed under the Creative Commons Attribution License, which permits unrestricted use, distribution, and reproduction in any medium, provided the original work is properly cited.

This paper investigates the existence of solutions and nonnegative solutions for prescribed variable exponent mean curvature impulsive system initialized boundary value problems. The proof of our main result is based upon Leray-Schauder's degree. The sufficient conditions for the existence of solutions and nonnegative solutions have been given.

\section{Introduction}

The theory of impulsive differential equations describes processes which experience a sudden change of their state at certain moments. On the Laplacian impulsive differential equations boundary value problems, there are many results (see [1-5]). Because of the nonlinearity of $p$-Laplacian, the results about $p$-Laplacian impulsive differential equations boundary value problems are rare (see [6]). In $[7,8]$, the authors discussed the existence of solutions of $p(r)$-Laplacian system impulsive boundary value problems. Recently, the existence and asymptotic behavior of solutions of curvature equations have been studied extensively (see [9-15]). In [16], the authors generalized the usual mean curvature systems to variable exponent mean curvature systems. In this paper, we consider the existence of 
solutions and nonnegative solutions for the prescribed variable exponent mean curvature system

$$
-\left(\varphi\left(r, u^{\prime}\right)\right)^{\prime}+f\left(r, u, u^{\prime}\right)=0, \quad r \in(0, T), r \neq r_{i}
$$

where $u:[0, T] \rightarrow \mathbb{R}^{N}$, with the following impulsive initialized boundary value conditions

$$
\begin{gathered}
\lim _{r \rightarrow r_{i}^{+}} u(r)-\lim _{r \rightarrow r_{i}^{-}} u(r)=A_{i}\left(\lim _{r \rightarrow r_{i}^{-}} u(r), \lim _{r \rightarrow r_{i}^{-}} u^{\prime}(r)\right), \quad i=1, \ldots, k, \\
\lim _{r \rightarrow r_{i}^{+}} \varphi\left(r, u^{\prime}(r)\right)-\lim _{r \rightarrow r_{i}^{-}} \varphi\left(r, u^{\prime}(r)\right)=B_{i}\left(\lim _{r \rightarrow r_{i}^{-}} u(r), \lim _{r \rightarrow r_{i}^{-}} u^{\prime}(r)\right), \quad i=1, \ldots, k, \\
u^{\prime}(0)=u(0)=0,
\end{gathered}
$$

where

$$
\varphi(r, x)=\frac{|x|^{p(r)-1} x}{\left(1+|x|^{q(r) p(r)}\right)^{1 / q(r)}}, \quad \forall r \in[0, T], x \in \mathbb{R}^{N},
$$

$p, q \in C\left([0, T], \mathbb{R}^{+}\right)$are absolutely continuous, where $p, q$ satisfy $p(r) \geq 1, q(r) \geq 1$, $-\left(\varphi\left(r, u^{\prime}\right)\right)^{\prime}$ is called the variable exponent mean curvature operator, $0<r_{1}<r_{2}<\cdots<$ $r_{k}<T$ and $A_{i}, B_{i} \in C\left(\mathbb{R}^{N} \times \mathbb{R}^{N}, \mathbb{R}^{N}\right)$.

For any $v \in \mathbb{R}^{N}, v^{j}$ will denote the $j$ th component of $v$; the inner product in $\mathbb{R}^{N}$ will be denoted by $\langle\cdot, \cdot\rangle ;|\cdot|$ will denote the absolute value and the Euclidean norm on $\mathbb{R}^{N}$. Denote that $J=[0, T], J^{\prime}=[0, T] \backslash\left\{r_{0}, r_{1}, \ldots, r_{k+1}\right\}$, and $J_{0}=\left[r_{0}, r_{1}\right], J_{i}=\left(r_{i}, r_{i+1}\right], i=1, \ldots, k$, where $r_{0}=0, r_{k+1}=T$. Denote that $J_{i}^{o}$ is the interior of $J_{i}, i=0,1, \ldots, k$. Let

$$
\begin{aligned}
& \operatorname{PC}\left(J, \mathbb{R}^{N}\right)=\left\{\begin{array}{l|l}
x: J \longrightarrow \mathbb{R}^{N} & \begin{array}{l}
x \in C\left(J_{i}, \mathbb{R}^{N}\right), i=0,1, \ldots, k, \\
\lim _{r \rightarrow r_{i}^{+}} x(r) \text { exists for } i=1, \ldots, k
\end{array}
\end{array}\right\}, \\
& \operatorname{PC}^{1}\left(J, \mathbb{R}^{N}\right)=\left\{x \in \operatorname{PC}\left(J, \mathbb{R}^{N}\right) \mid \begin{array}{c}
x^{\prime} \in C\left(J_{i}^{o}, \mathbb{R}^{N}\right), \\
\lim _{r \rightarrow r_{i}^{+}} x^{\prime}(r) \text { and } \lim _{r \rightarrow r_{i+1}^{-}} x^{\prime}(r) \text { exist for } i=0,1, \ldots, k
\end{array}\right\} .
\end{aligned}
$$

For any $u(r)=\left(u^{1}(r), \ldots, u^{N}(r)\right) \in \operatorname{PC}\left(J, \mathbb{R}^{N}\right)$, denote that $\left|u^{i}\right|_{0}=\sup \left\{\left|u^{i}(r)\right| \mid r \in J^{\prime}\right\}$. Obviously, $\operatorname{PC}\left(J, \mathbb{R}^{N}\right)$ is a Banach space with the norm $\|u\|_{0}=\left(\sum_{i=1}^{N}\left|u^{i}\right|_{0}^{2}\right)^{1 / 2}$, and $\operatorname{PC}^{1}\left(J, \mathbb{R}^{N}\right)$ is a Banach space with the norm $\|u\|_{1}=\|u\|_{0}+\left\|u^{\prime}\right\|_{0}$. In the following, $\operatorname{PC}\left(J, \mathbb{R}^{N}\right)$ and $\operatorname{PC}^{1}\left(J, \mathbb{R}^{N}\right)$ will be simply denoted by PC and $\mathrm{PC}^{1}$, respectively. Denote that $L^{1}=L^{1}\left(J, \mathbb{R}^{N}\right)$, and the norm in $L^{1}$ is $\|u\|_{L^{1}}=\left[\sum_{i=1}^{N}\left(\int_{0}^{T}\left|u^{i}(r)\right| d r\right)^{2}\right]^{1 / 2}$.

The study of differential equations and variational problems with variable exponent conditions is a new and interesting topic. For the applied background on this kind of problems we refer to [17-19]. Many results have been obtained on this kind of problems, 
for example, [20-35]. If $p(r) \equiv p$ (a constant) and $q(r) \equiv q$ (a constant), then (1.1) is the wellknown mean curvature system. Since problems with variable exponent growth conditions are more complex than those with constant exponent growth conditions, many methods and results for the latter are invalid for the former; for example, if $\Omega \subset \mathbb{R}^{n}$ is a bounded domain, the Rayleigh quotient

$$
\lambda_{p(x)}=\inf _{u \in W_{0}^{1, p(x)}(\Omega) \backslash\{0\}} \frac{\int_{\Omega}(1 / p(x))|\nabla u|^{p(x)} d x}{\int_{\Omega}(1 / p(x))|u|^{p(x)} d x}
$$

is zero in general, and only under some special conditions $\lambda_{p(x)}>0$ (see [25]), but the fact that $\lambda_{p}>0$ is very important in the study of $p$-Laplacian problems.

In this paper, we investigate the existence of solutions for the prescribed variable exponent mean curvature impulsive differential system initialized boundary value problems; the proof of our main result is based upon Leray-Schauder's degree. This paper was motivated by $[6,13,36]$.

Let $N \geq 1$, then the function $f: J \times \mathbb{R}^{N} \times \mathbb{R}^{N} \rightarrow \mathbb{R}^{N}$ is assumed to be Caratheodory; by this we mean that

(i) for almost every $t \in J$ the function $f(t, \cdot, \cdot)$ is continuous,

(ii) for each $(x, y) \in \mathbb{R}^{N} \times \mathbb{R}^{N}$ the function $f(\cdot, x, y)$ is measurable on $J$,

(iii) for each $R>0$ there is a $\beta_{R} \in L^{1}(J, \mathbb{R})$ such that, for almost every $t \in J$ and every $(x, y) \in \mathbb{R}^{N} \times \mathbb{R}^{N}$ with $|x| \leq R,|y| \leq R$, one has

$$
|f(t, x, y)| \leq \beta_{R}(t)
$$

We say a function $u: J \rightarrow \mathbb{R}^{N}$ is a solution of (1.1) if $u \in \mathrm{PC}^{1}$ with $\varphi\left(r, u^{\prime}\right)$ absolutely continuous on $J_{i}^{o}, i=0,1, \ldots, k$, which satisfies (1.1) a.e. on $J$.

This paper is divided into three sections; in the second section, we present some preliminary. Finally, in the third section, we give the existence of solutions and nonnegative solutions for system (1.1)-(1.4).

\section{Preliminary}

In this section, we will do some preparation.

Lemma 2.1 (see [16]). $\varphi$ is a continuous function and satisfies the following.

(i) For any $r \in J, \varphi(r, \cdot)$ is strictly monotone, that is,

$$
\left\langle\varphi\left(r, x_{1}\right)-\varphi\left(r, x_{2}\right), x_{1}-x_{2}\right\rangle>0, \quad \text { for any } x_{1}, x_{2} \in \mathbb{R}^{N}, x_{1} \neq x_{2}
$$

(ii) For any fixed $r \in J, \varphi(r, \cdot)$ is a homeomorphism from $\mathbb{R}^{N}$ to

$$
E=\left\{x \in \mathbb{R}^{N}|| x \mid<1\right\}
$$


For any $r \in J$, denote by $\varphi^{-1}(r, \cdot)$ the inverse operator of $\varphi(r, \cdot)$, then

$$
\varphi^{-1}(r, x)=\left(1-|x|^{q(r)}\right)^{-1 / p(r) q(r)}|x|^{1 / p(r)-1} x, \quad \text { for } x \in E \backslash\{0\}, \varphi^{-1}(r, 0)=0 .
$$

Let one now consider the following simple problem:

$$
\left(\varphi\left(r, u^{\prime}(r)\right)\right)^{\prime}=h(r), \quad r \in(0, T), r \neq r_{i}
$$

with the following impulsive boundary value conditions:

$$
\begin{gathered}
\lim _{r \rightarrow r_{i}^{+}} u(r)-\lim _{r \rightarrow r_{i}^{-}} u\left(r_{i}\right)=a_{i}, \quad i=1, \ldots, k, \\
\lim _{r \rightarrow r_{i}^{+}} \varphi\left(r, u^{\prime}(r)\right)-\lim _{r \rightarrow r_{i}^{-}} \varphi\left(r, u^{\prime}(r)\right)=b_{i}, \quad i=1, \ldots, k, \\
u^{\prime}(0)=u(0)=0,
\end{gathered}
$$

where $a_{i}, b_{i} \in \mathbb{R}^{N}, \sum_{i=1}^{k}\left|b_{i}\right|<1 ; h \in L^{1}$.

Obviously, $u^{\prime}(0)=0$ implies that $\varphi\left(0, u^{\prime}(0)\right)=0$. If $u$ is a solution of (2.4) with (2.5), by integrating (2.4) from 0 to $r$, then one finds that

$$
\varphi\left(r, u^{\prime}(r)\right)=\sum_{r_{i}<r} b_{i}+\int_{0}^{r} h(t) d t, \quad \forall r \in J^{\prime}
$$

Define $a=\left(a_{1}, \ldots, a_{k}\right) \in \mathbb{R}^{k N}, b=\left(b_{1}, \ldots, b_{k}\right) \in \mathbb{R}^{k N}$, and operator $F: L^{1} \rightarrow P C$ as

$$
F(h)(r)=\int_{0}^{r} h(t) d t, \quad \forall h \in L^{1}
$$

From the definition of $\varphi$, one can see that

$$
\sup _{r \in J}\left|\sum_{r_{i}<r} b_{i}+F(h)(r)\right|<1 .
$$

Denote that

$$
D_{b}=\left\{h \in L^{1}\left|\sup _{r \in J}\right| \sum_{r_{i}<r} b_{i}+F(h)(r) \mid<1\right\} .
$$

By (2.6), one has

$$
u(r)=\sum_{r_{i}<r} a_{i}+F\left\{\varphi^{-1}\left[r,\left(\sum_{r_{i}<r} b_{i}+F(h)\right)\right]\right\}(r), \quad \forall r \in J .
$$


Journal of Inequalities and Applications

Denote that $W=\mathbb{R}^{2 k N} \times L^{1}$ with the norm

$$
\|w\|_{W}=\sum_{i=1}^{k}\left|a_{i}\right|+\sum_{i=1}^{k}\left|b_{i}\right|+\|h\|_{L^{1}}, \quad \forall w=(a, b, h) \in W,
$$

then $W$ is a Banach space.

Let one define the nonlinear operator $K_{b}: D_{b} \rightarrow P C^{1}$ as

$$
K_{b}(h)(r)=F\left\{\varphi^{-1}\left[r,\left(\sum_{r_{i}<r} b_{i}+F(h)\right)\right]\right\}(r), \quad \forall r \in J .
$$

Lemma 2.2. The operator $K_{b}$ is continuous and sends closed equiintegrable subsets of $D_{b}$ into relatively compact sets in $P C^{1}$.

Proof. It is easy to check that $K_{b}(h)(\cdot) \in \mathrm{PC}^{1}$, for all $h \in D_{b}$. Since

$$
K_{b}(h)^{\prime}(t)=\varphi^{-1}\left\{t,\left[\sum_{r_{i}<t} b_{i}+F(h)\right]\right\}, \quad \forall t \in J,
$$

it is easy to check that $K_{b}$ is a continuous operator from $D_{b}$ to $\mathrm{PC}^{1}$.

Let now $\bar{U}$ be a closed equiintegrable set in $D_{b}$, then there exists $\eta \in L^{1}$, such that, for any $u \in \bar{U}$,

$$
|u(t)| \leq \eta(t) \quad \text { a.e. on } J
$$

We want to show that $\overline{K_{b}(U)} \subset \mathrm{PC}^{1}$ is a compact set.

Let $\left\{u_{n}\right\}$ is a sequence in $K_{b}(\bar{U})$, then there exists a sequence $\left\{h_{n}\right\} \in \bar{U}$ such that $u_{n}=K_{b}\left(h_{n}\right)$. For any $t_{1}, t_{2} \in J$, we have that

$$
\left|F\left(h_{n}\right)\left(t_{1}\right)-F\left(h_{n}\right)\left(t_{2}\right)\right| \leq\left|\int_{t_{1}}^{t_{2}} \eta(t) d t\right| .
$$

Hence the sequence $\left\{F\left(h_{n}\right)\right\}$ is uniformly bounded and equicontinuous. By AscoliArzela theorem, there exists a subsequence of $\left\{F\left(h_{n}\right)\right\}$ (which we rename the same) that is convergent in PC. Then the sequence

$$
\varphi\left(t, K_{b}\left(h_{n}\right)^{\prime}(t)\right)=\sum_{r_{i}<t} b_{i}+F\left(h_{n}\right)
$$

is convergent according to the norm in PC. Since

$$
K_{b}\left(h_{n}\right)(t)=F\left\{\varphi^{-1}\left[t,\left(\sum_{r_{i}<t} b_{i}+F\left(h_{n}\right)\right)\right]\right\}(t), \quad \forall t \in J
$$


according to the continuity of $\varphi^{-1}$, we can see that $K_{b}\left(h_{n}\right)$ is convergent in PC. Thus we conclude that $\left\{u_{n}\right\}$ is convergent in $\mathrm{PC}^{1}$. This completes the proof.

We denote that $N_{f}(u): \mathrm{PC}^{1} \rightarrow L^{1}$ is the Nemytski operator associated to $f$ defined by

$$
N_{f}(u)(r)=f\left(r, u(r), u^{\prime}(r)\right), \quad \text { a.e. on } J
$$

Define $K: \mathrm{PC}^{1} \rightarrow \mathrm{PC}^{1}$ as

$$
K(u)(r)=F\left\{\varphi^{-1}\left[r,\left(\sum_{r_{i}<r} B_{i}+F\left(N_{f}(u)\right)\right)\right]\right\}(r), \quad \forall r \in J,
$$

where $B_{i}=B_{i}\left(\lim _{r \rightarrow r_{i}^{-}} u(r), \lim _{r \rightarrow r_{i}^{-}} u^{\prime}(r)\right)$.

Lemma 2.3. $u$ is a solution of (1.1)-(1.4) if and only if $u$ is a solution of the following abstract equation:

$$
u=\sum_{r_{i}<r} A_{i}+K(u)
$$

where $A_{i}=A_{i}\left(\lim _{r \rightarrow r_{i}^{-}} u(r), \lim _{r \rightarrow r_{i}^{-}} u^{\prime}(r)\right), B_{i}=B_{i}\left(\lim _{r \rightarrow r_{i}^{-}} u(r)\right.$, and $\left.\lim _{r \rightarrow r_{i}^{-}} u^{\prime}(r)\right)$.

Proof. (i) If $u$ is a solution of (1.1)-(1.4), since $u^{\prime}(0)=0$ implies that $\varphi\left(0, u^{\prime}(0)\right)=0$, by integrating (1.1) from 0 to $r$, then we find that

$$
\varphi\left(r, u^{\prime}(r)\right)=\sum_{r_{i}<r} B_{i}+\int_{0}^{r} f\left(t, u, u^{\prime}\right) d t, \quad \forall r \in J^{\prime}
$$

Thus

$$
u=\sum_{r_{i}<r} A_{i}+K(u)
$$

Hence $u$ is a solution of (2.20). we have

(ii) If $u$ is a solution of (2.20), then it is easy to see that (1.2) is satisfied. Let $r=0$, then

$$
u(0)=0
$$

From (2.20) we also have

$$
\begin{gathered}
\varphi\left(r, u^{\prime}\right)=\sum_{r_{i}<r} B_{i}+F\left(N_{f}(u)\right)(r), \quad r \in(0, T), r \neq r_{i}, \\
\left(\varphi\left(r, u^{\prime}\right)\right)^{\prime}=f\left(r, u, u^{\prime}\right), \quad r \in(0, T), r \neq r_{i} .
\end{gathered}
$$


Journal of Inequalities and Applications

From (2.24), we can see that (1.3) is satisfied. Let $r=0$, from (2.24), then we have

$$
\varphi\left(0, u^{\prime}(0)\right)=0 .
$$

Since $\varphi(r, x)=|x|^{p(r)-1} x /\left(1+|x|^{q(r) p(r)}\right)^{1 / q(r)}=0$, we must have $x=0$; thus,

$$
u^{\prime}(0)=0 \text {. }
$$

Hence $u$ is a solution of (1.1)-(1.4). This completes the proof.

\section{Main Results and Proofs}

In this section, we will apply Leray-Schauder's degree to deal with the existence of solutions for (1.1)-(1.4).

We assume that

$$
\left(\mathrm{H}_{0}\right) \sum_{i=1}^{k}\left|B_{i}(u, v)\right|<1 / 2 \text {, for all }(u, v) \in \mathbb{R}^{N} \times \mathbb{R}^{N} .
$$

Theorem 3.1. If $\left(H_{0}\right)$ is satisfied, then $0 \in \Omega$ is an open bounded set in $P C^{1}$ such that the following conditions hold.

$\left(1^{0}\right)$ For any $u \in \bar{\Omega}$, the mapping $r \rightarrow f\left(r, u, u^{\prime}\right)$ belongs to $\left\{v \in L^{1} \mid\|v\|_{L^{1}}<1 / 3\right\}$.

$\left(2^{0}\right)$ For each $\lambda \in(0,1)$, the problem

$$
\begin{gathered}
\left(\varphi\left(r, u^{\prime}\right)\right)^{\prime}=\lambda f\left(r, u, u^{\prime}\right), \quad r \in(0, T), r \neq r_{i}, \\
\lim _{r \rightarrow r_{i}^{+}} u(r)-\lim _{r \rightarrow r_{i}^{-}} u(r)=\lambda A_{i}\left(\lim _{r \rightarrow r_{i}^{-}} u(r), \lim _{r \rightarrow r_{i}^{-}} u^{\prime}(r)\right), \quad i=1, \ldots, k, \\
\lim _{r \rightarrow r_{i}^{+}} \varphi\left(r, u^{\prime}(r)\right)-\lim _{r \rightarrow r_{i}^{-}} \varphi\left(r, u^{\prime}(r)\right)=\lambda B_{i}\left(\lim _{r \rightarrow r_{i}^{-}} u(r), \lim _{r \rightarrow r_{i}^{-}} u^{\prime}(r)\right), \quad i=1, \ldots, k, \\
u^{\prime}(0)=u(0)=0
\end{gathered}
$$

has no solution on $\partial \Omega$.

Then (1.1)-(1.4) has at least one solution on $\bar{\Omega}$.

Proof. Denote that

$$
A_{i}=A_{i}\left(\lim _{r \rightarrow r_{i}^{-}} u(r), \lim _{r \rightarrow r_{i}^{-}} u^{\prime}(r)\right), \quad B_{i}=B_{i}\left(\lim _{r \rightarrow r_{i}^{-}} u(r), \lim _{r \rightarrow r_{i}^{-}} u^{\prime}(r)\right) .
$$

For any $\lambda \in[0,1]$, define $K_{\curlywedge}: \mathrm{PC}^{1} \rightarrow \mathrm{PC}^{1}$ as

$$
K_{\lambda}(u)(r)=F\left\{\varphi^{-1}\left[r,\left(\sum_{r_{i}<r} \lambda B_{i}+F\left(\lambda N_{f}(u)\right)\right)\right]\right\}(r), \quad \forall r \in J .
$$

Denote that $\Psi(u, \lambda):=\lambda \sum_{r_{i}<r} A_{i}+K_{\curlywedge}(u)$. 
We know that (1.1)-(1.4) has the same solutions of

$$
u=\Psi(u, 1)=\sum_{r_{i}<r} A_{i}+K_{1}(u)
$$

Since $f$ is Caratheodory, it is easy to see that $N_{f}(\cdot)$ is continuous and sends bounded sets into equiintegrable sets. According to Lemma 2.2, we can conclude that $\Psi$ is compact continuous on $\bar{\Omega}$ for any $\lambda \in[0,1]$. We assume that for $\lambda=1$ (3.4) does not have a solution on $\partial \Omega$; otherwise, we complete the proof. Now from hypothesis $\left(2^{0}\right)$, it follows that (3.4) has no solution for $(u, \lambda) \in \partial \Omega \times(0,1]$.

When $\lambda=0$, (3.1) is equivalent to the following usual problem:

$$
-\left(\varphi\left(r, u^{\prime}\right)\right)^{\prime}=0, \quad u^{\prime}(0)=u(0)=0,
$$

where obviously 0 is the unique solution to this problem.

Since $0 \in \Omega$, we have proved that (3.4) has no solution $(u, \lambda)$ on $\partial \Omega \times[0,1]$, then we get that, for each $\lambda \in[0,1]$, Leray-Schauder's degree $d_{\mathrm{LS}}[I-\Psi(\cdot, \lambda), \Omega, 0]$ is well defined. From the homotopy invariant property of that degree, we have

$$
d_{\mathrm{LS}}[I-\Psi(u, 1), \Omega, 0]=d_{\mathrm{LS}}[I-\Psi(u, 0), \Omega, 0]=1 .
$$

This completes the proof.

In the following, we will give an application of Theorem 3.1.

Denote that $\sigma=4 T /(4 T+1)$ and

$$
\Omega_{\varepsilon}=\left\{u \in \mathrm{PC}^{1} \mid \max _{1 \leq j \leq N}\left(\left|u^{j}\right|_{0}+\left|\left(u^{j}\right)^{\prime}\right|_{0}\right)<\varepsilon\right\}
$$

Obviously, $\Omega_{\varepsilon}$ is an open subset of $\mathrm{PC}^{1}$.

Assume that

$\left(\mathrm{H}_{1}\right) f(r, u, v)=\delta g(r, u, v)$, where $\delta$ is a positive parameter, and $g$ is Caratheodory.

$\left(\mathrm{H}_{2}\right) \sum_{i=1}^{k}\left|A_{i}(u, v)\right| \leq(\sigma / 2) \varepsilon, \sum_{i=1}^{k}\left|B_{i}(u, v)\right| \leq\left\{\min _{r \in I}|\varepsilon /(4 T+1)|^{p(r)} / 2\left(1+|N \varepsilon|^{q(r) p(r)}\right)^{1 / q(r)}\right.$, $1 / 2\}$, for all $(u, v) \in \mathbb{R}^{N} \times \mathbb{R}^{N}$.

Theorem 3.2. If $\left(H_{1}\right)-\left(H_{2}\right)$ are satisfied, then problem (1.1)-(1.4) has at least one solution on $\overline{\Omega_{\varepsilon}}$, when positive parameter $\delta$ is small enough.

Proof. Let one consider the problem

$$
u=\Psi(u, \lambda)=\lambda \sum_{r_{i}<r} A_{i}+K_{\curlywedge}(u)
$$

where $K_{\curlywedge}(\cdot)$ is defined in (3.3). 
Obviously, $u$ is a solution of (1.1)-(1.4) if and only if $u$ is a solution of the abstract equation (3.8) when $\lambda=1$. We only need to prove that the conditions of Theorem 3.1 are satisfied.

$\left(1^{0}\right)$ When positive parameter $\delta$ is small enough, for any $u \in \overline{\Omega_{\varepsilon}}$, we can see that the mapping $r \mapsto \delta g\left(r, u, u^{\prime}\right)$ belongs to $\left\{u \in L^{1} \mid\|u\|_{L^{1}}<1 / 3\right\}$.

$\left(2^{0}\right)$ We shall prove that for each $\lambda \in(0,1)$ the problem

$$
\begin{gathered}
\left(\varphi\left(r, u^{\prime}\right)\right)^{\prime}=\lambda f\left(r, u, u^{\prime}\right), \quad r \in(0, T), r \neq r_{i}, \\
\lim _{r \rightarrow r_{i}^{+}} u(r)-\lim _{r \rightarrow r_{i}^{-}} u(r)=\lambda A_{i}\left(\lim _{r \rightarrow r_{i}^{-}} u(r), \lim _{r \rightarrow r_{i}^{-}} u^{\prime}(r)\right), i=1, \ldots, k, \\
\lim _{r \rightarrow r_{i}^{+}} \varphi\left(r, u^{\prime}(r)\right)-\lim _{r \rightarrow r_{i}^{-}} \varphi\left(r, u^{\prime}(r)\right)=\lambda B_{i}\left(\lim _{r \rightarrow r_{i}^{-}} u(r), \lim _{r \rightarrow r_{i}^{-}} u^{\prime}(r)\right), \quad i=1, \ldots, k, \\
u^{\prime}(0)=u(0)=0
\end{gathered}
$$

has no solution on $\partial \Omega_{\varepsilon}$.

If it is false, then there exists a $\lambda \in(0,1)$ and $u \in \partial \Omega_{\varepsilon}$ is a solution of (3.8). Then there exists an $j \in\{1, \ldots, N\}$ such that $\left|u^{j}\right|_{0}+\left|\left(u^{j}\right)^{\prime}\right|_{0}=\varepsilon$.

(i) Suppose that $\left|u^{j}\right|_{0} \geq \sigma \varepsilon$, then $\left|\left(u^{j}\right)^{\prime}\right|_{0} \leq(1-\sigma) \varepsilon$. For any $r \in J$, since $u(0)=0$, according to $\left(\mathrm{H}_{2}\right)$ and (1.2), we have

$$
\begin{aligned}
\left|u^{j}(r)\right| & =\left|u^{j}(r)-u^{j}(0)\right|=\left|\int_{0}^{r}\left(u^{j}\right)^{\prime}(t) d t+\sum_{0<r_{i}<r} A_{i}\right| \\
& \leq \int_{0}^{T}\left|\left(u^{j}\right)^{\prime}(t)\right| d t+\sum_{0<r_{i}<r}\left|A_{i}\right| \\
& \leq \int_{0}^{T}(1-\sigma) \varepsilon d r+\sum_{i=1}^{k}\left|A_{i}\right| \\
& \leq \frac{\sigma}{4} \varepsilon+\frac{\sigma}{2} \varepsilon=\frac{3 \sigma}{4} \varepsilon .
\end{aligned}
$$

It is a contradiction.

(ii) Suppose that $\left|u^{j}\right|_{0}<\sigma \varepsilon,(1-\sigma) \varepsilon<\left|\left(u^{j}\right)^{\prime}\right|_{0} \leq \varepsilon$. This implies that $\left|\left(u^{j}\right)^{\prime}\left(r_{*}\right)\right|>(1-\sigma) \varepsilon=$ $(1 /(4 T+1)) \varepsilon$ for some $r_{*} \in J$.

Denote that

$$
\varphi^{j}\left(r, u^{\prime}\right)(r)=\frac{\left|u^{\prime}\right|^{p(r)-1}\left(u^{j}\right)^{\prime}(r)}{\left(1+\left|u^{\prime}\right|^{q(r) p(r)}\right)^{1 / q(r)}} .
$$


Since $u^{\prime}(0)=0$, we have

$$
\varphi^{j}\left(r_{*}, u^{\prime}\right)\left(r_{*}\right)=\lambda \int_{0}^{r_{*}} f^{j}\left(r, u, u^{\prime}\right) d r+\lambda \sum_{0<r_{i}<r_{*}} B_{i}^{j}
$$

According to $\left(\mathrm{H}_{1}\right)-\left(\mathrm{H}_{2}\right)$, when positive parameter $\delta$ is small enough, we have

$$
\begin{aligned}
\frac{|\varepsilon /(4 T+1)|^{p\left(r_{*}\right)}}{\left(1+|N \varepsilon|^{q\left(r_{*}\right) p\left(r_{*}\right)}\right)^{1 / q\left(r_{*}\right)}} & \leq\left|\varphi^{j}\left(r_{*}, u^{\prime}\right)\left(r_{*}\right)\right| \leq \lambda \int_{0}^{r_{*}}\left|f^{j}\left(r, u, u^{\prime}\right)\right| d r+\lambda\left|\sum_{0<r_{i}<r_{*}} B_{i}^{j}\right| \\
& \leq \delta \int_{0}^{T} \beta_{N \varepsilon}(r) d r+\sum_{i=1}^{k}\left|B_{i}\right|<\frac{|\varepsilon /(4 T+1)|^{p\left(r_{*}\right)}}{\left(1+|N \varepsilon|^{q\left(r_{*}\right) p\left(r_{*}\right)}\right)^{1 / q\left(r_{*}\right)}}
\end{aligned}
$$

It is a contradiction.

Summarizing this argument, for each $\lambda \in(0,1)$, the problem (3.8) with (1.4) has no solution on $\partial \Omega_{\varepsilon}$.

Since $0 \in \Omega_{\varepsilon}$ and 0 is the unique solution of $u=\Psi(u, 0)$, then the Leray-Schauder's degree

$$
d_{\mathrm{LS}}[I-\Psi(\cdot, 0), \Omega, 0]=1 \neq 0 .
$$

This completes the proof.

In the following, we will discuss the existence of nonnegative solutions of (1.1)-(1.4). For any $x=\left\{x^{1}, \ldots, x^{N}\right\} \in \mathbb{R}^{N}$, the notation $x \geq 0(x>0)$ means that $x^{l} \geq 0\left(x^{l}>0\right)$ for every $l \in\{1, \ldots, N\}$.

Assume the following

$\left(\mathrm{H}_{3}\right) f(r, u, v)=\delta g(r, u, v)$, where $\delta$ is a positive parameter, and

$$
g(r, u, v)=\tau(r)\left[|u|^{q_{1}(r)-1} u+\mu(r)|v|^{q_{2}(r)-1} v\right]+\gamma(r)
$$

where $q_{1}, q_{2} \in C(J, \mathbb{R}), 0 \leq q_{1}(r)$, and $0 \leq q_{2}(r)$, for all $r \in J$.

$\left(\mathrm{H}_{4}\right) A_{i}^{j}(x, y) y^{j} \geq 0$, and $B_{i}^{j}(x, y) y^{j} \geq 0$, for all $x, y \in \mathbb{R}^{N}, i=1, \ldots, k, j=1, \ldots, N$.

$\left(\mathrm{H}_{5}\right) \mu, \tau \in C\left(J, \mathbb{R}^{+}\right)$.

$\left(\mathrm{H}_{6}\right) \gamma=\left(\gamma^{1}, \ldots, \gamma^{N}\right) \in C\left(J, \mathbb{R}^{N}\right)$ satisfies $\gamma(0)>0, \int_{0}^{t} \gamma(s) d s \geq 0$, for all $t \in[0, T]$.

Theorem 3.3. If $\left(\mathrm{H}_{2}\right)-\left(\mathrm{H}_{6}\right)$ are satisfied, then the problem (1.1)-(1.4) has a nonnegative solution, when positive parameter $\delta$ is small enough. 
Proof. From Theorem 3.2, we can get the existence of solutions of (1.1)-(1.4). If $u$ is a solution of (1.1)-(1.4), according to (1.4) and $\left(\mathrm{H}_{6}\right)$, then we have

$$
f\left(0, u(0), u^{\prime}(0)\right)=\delta \gamma(0)>0
$$

Obviously

$$
\begin{aligned}
& \varphi\left(r, u^{\prime}\right)(r)=\int_{0}^{r} \delta\left\{\tau(r)\left[|u|^{q_{1}(r)-1} u+\mu(r)\left|u^{\prime}\right|^{q_{2}(r)-1} u^{\prime}\right]+\gamma(r)\right\} d s, \quad \forall r \in\left[0, r_{1}\right) . \\
& \text { When } r \rightarrow 0^{+}, \text {we have }
\end{aligned}
$$

$$
\tau(r)\left[|u|^{q_{1}(r)-1} u+\mu(r)\left|u^{\prime}\right|^{q_{2}(r)-1} u^{\prime}\right]+\gamma(r)>0,
$$

then we can see that there exists a $\xi \in\left(0, r_{1}\right)$ such that $\varphi\left(r, u^{\prime}\right)(r)>0$ when $r \in(0, \xi)$. Thus $u^{\prime}(r)>0$ for any $r \in(0, \xi)$. Thus $u(r)$ is increasing in $(0, \xi)$, that is, $u\left(\eta_{2}\right) \geq u\left(\eta_{1}\right)$ for any $\eta_{1}, \eta_{2} \in(0, \xi)$ with $\eta_{1}<\eta_{2}$. Since $u(0)=0$, it is easy to see that $u(r)>0$ for any $r \in(0, \xi)$. From (3.17) and $\left(\mathrm{H}_{5}\right)$, we can easily see that

$$
\begin{gathered}
u(r)>0, \quad u^{\prime}(r)>0, \quad \forall r \in\left(0, r_{1}\right), \\
\lim _{r \rightarrow r_{1}^{-}} u(r)>0, \quad \lim _{r \rightarrow r_{1}^{-}} u^{\prime}(r)>0 .
\end{gathered}
$$

From $\left(\mathrm{H}_{4}\right)$, we can see that

$$
\lim _{r \rightarrow r_{1}^{+}} u(r)>0, \quad \lim _{r \rightarrow r_{1}^{+}} u^{\prime}(r)>0 .
$$

Similarly, we can see that

$$
u(r)>0, \quad u^{\prime}(r)>0, \quad \forall r \in\left(r_{1}, r_{2}\right) .
$$

Repeating the step, we can see that

$$
u(r)>0, \quad u^{\prime}(r)>0, \quad \forall r \in J^{\prime} .
$$

Hence $u$ is nonnegative. This completes the proof.

\section{Acknowledgments}

This paper is partly supported by the National Science Foundation of China (10701066, 10926075 and 10971087), China Postdoctoral Science Foundation funded project (20090460969), the Natural Science Foundation of Henan Education Committee (2008-75565), and the Natural Science Foundation of Jiangsu Education Committee (08KJD110007). 


\section{References}

[1] J. Jiao, L. Chen, and L. Li, "Asymptotic behavior of solutions of second-order nonlinear impulsive differential equations," Journal of Mathematical Analysis and Applications, vol. 337, no. 1, pp. 458-463, 2008.

[2] L. Liu, L. Hu, and Y. Wu, "Positive solutions of two-point boundary value problems for systems of nonlinear second-order singular and impulsive differential equations," Nonlinear Analysis: Theory, Methods E Applications, vol. 69, no. 11, pp. 3774-3789, 2008.

[3] X. Liu and D. Guo, "Periodic boundary value problems for a class of second-order impulsive integrodifferential equations in Banach spaces," Journal of Mathematical Analysis and Applications, vol. 216, no. 1, pp. 284-302, 1997.

[4] J. Shen and W. Wang, "Impulsive boundary value problems with nonlinear boundary conditions," Nonlinear Analysis: Theory, Methods \& Applications, vol. 69, no. 11, pp. 4055-4062, 2008.

[5] M. Yao, A. Zhao, and J. Yan, "Periodic boundary value problems of second-order impulsive differential equations," Nonlinear Analysis: Theory, Methods E Applications, vol. 70, no. 1, pp. 262-273, 2009.

[6] A. Cabada and J. Tomeček, "Extremal solutions for nonlinear functional $\phi$-Laplacian impulsive equations," Nonlinear Analysis: Theory, Methods E Applications, vol. 67, no. 3, pp. 827-841, 2007.

[7] Q. Zhang, X. Liu, and Z. Qiu, "Existence of solutions for weighted $p(r)$-Laplacian impulsive system periodic-like boundary value problems," Nonlinear Analysis: Theory, Methods \& Applications, vol. 71, no. 9, pp. 3596-3611, 2009.

[8] Q. Zhang, Z. Qiu, and X. Liu, "Existence of solutions and nonnegative solutions for weighted $p(r)$-Laplacian impulsive system multi-point boundary value problems," Nonlinear Analysis: Theory, Methods \& Applications, vol. 71, no. 9, pp. 3814-3825, 2009.

[9] R. Filippucci, "Entire radial solutions of elliptic systems and inequalities of the mean curvature type," Journal of Mathematical Analysis and Applications, vol. 334, no. 1, pp. 604-620, 2007.

[10] A. Greco, "On the existence of large solutions for equations of prescribed mean curvature," Nonlinear Analysis: Theory, Methods \& Applications, vol. 34, no. 4, pp. 571-583, 1998.

[11] N. M. Ivochkina, "The Dirichlet problem for the curvature equation of order $m$," Leningrad Mathematical Journal, vol. 2, pp. 631-654, 1991.

[12] K. E. Lancaster and D. Siegel, "Existence and behavior of the radial limits of a bounded capillary surface at a corner," Pacific Journal of Mathematics, vol. 176, no. 1, pp. 165-194, 1996.

[13] H. Pan, "One-dimensional prescribed mean curvature equation with exponential nonlinearity," Nonlinear Analysis: Theory, Methods \& Applications, vol. 70, no. 2, pp. 999-1010, 2009.

[14] K. Takimoto, "Solution to the boundary blowup problem for $k$-curvature equation," Calculus of Variations and Partial Differential Equations, vol. 26, no. 3, pp. 357-377, 2006.

[15] N. S. Trudinger, "The Dirichlet problem for the prescribed curvature equations," Archive for Rational Mechanics and Analysis, vol. 111, no. 2, pp. 153-179, 1990.

[16] Q. Zhang, Z. Qiu, and X. Liu, "Existence of solutions for prescribed variable exponent mean curvature system boundary value problems," Nonlinear Analysis: Theory, Methods E Applications, vol. 71, no. 7-8, pp. 2964-2975, 2009.

[17] Y. Chen, S. Levine, and M. Rao, "Variable exponent, linear growth functionals in image restoration," SIAM Journal on Applied Mathematics, vol. 66, no. 4, pp. 1383-1406, 2006.

[18] M. Růžička, Electrorheological Fluids: Modeling and Mathematical Theory, vol. 1748 of Lecture Notes in Mathematics, Springer, Berlin, Germany, 2000.

[19] V. V. Zhikov, "Averaging of functionals of the calculus of variations and elasticity theory," Mathematics of the USSR. Izvestiya, vol. 29, pp. 33-36, 1987.

[20] X.-L. Fan, H.-Q. Wu, and F.-Z. Wang, “Hartman-type results for $p(t)$-Laplacian systems," Nonlinear Analysis: Theory, Methods \& Applications, vol. 52, no. 2, pp. 585-594, 2003.

[21] X.-L. Fan and D. Zhao, "Regularity of minimum points of variational integrals with continuous $p(x)$ growth conditions," Chinese Annals of Mathematics. Series A, vol. 17, no. 5, pp. 557-564, 1996.

[22] X.-L. Fan and D. Zhao, "On the spaces $L^{p(x)}(\Omega)$ and $W^{m, p(x)}(\Omega)$," Journal of Mathematical Analysis and Applications, vol. 263, no. 2, pp. 424-446, 2001.

[23] X.-L. Fan and Q.-H. Zhang, "Existence of solutions for $p(x)$-Laplacian Dirichlet problem," Nonlinear Analysis: Theory, Methods E Applications, vol. 52, no. 8, pp. 1843-1852, 2003.

[24] X.-L. Fan, Y. Z. Zhao, and Q. H. Zhang, "A strong maximum principle for $p(x)$-Laplace equations," Chinese Journal of Contemporary Mathematics, vol. 24, no. 4, pp. 495-500, 2003. 
[25] X.-L. Fan, Q. H. Zhang, and D. Zhao, "Eigenvalues of $p(x)$-Laplacian Dirichlet problem," Journal of Mathematical Analysis and Applications, vol. 302, no. 2, pp. 306-317, 2005.

[26] A. El Hamidi, "Existence results to elliptic systems with nonstandard growth conditions," Journal of Mathematical Analysis and Applications, vol. 300, no. 1, pp. 30-42, 2004.

[27] O. Kováčik and J. Rákosník, "On spaces $L^{p(x)}(\Omega)$ and $W^{k, p(x)}(\Omega)$," Czechoslovak Mathematical Journal, vol. 41, no. 4, pp. 592-618, 1991.

[28] P. Marcellini, "Regularity and existence of solutions of elliptic equations with $(p, q)$-growth conditions," Journal of Differential Equations, vol. 90, no. 1, pp. 1-30, 1991.

[29] M. Mihăilescu and V. Rădulescu, "Continuous spectrum for a class of nonhomogeneous differential operators," Manuscripta Mathematica, vol. 125, no. 2, pp. 157-167, 2008.

[30] J. Musielak, Orlicz Spaces and Modular Spaces, vol. 1034 of Lecture Notes in Mathematics, Springer, Berlin, Germany, 1983.

[31] S. G. Samko, "Density $C_{0}^{\infty}\left(\mathbb{R}^{N}\right)$ in the generalized Sobolev spaces $W^{m, p(x)}\left(\mathbb{R}^{N}\right)$," Doklady Akademii Nauk. Rossiǔskaya Akademiya Nauk, vol. 369, no. 4, pp. 451-454, 1999.

[32] Q. H. Zhang, "A strong maximum principle for differential equations with nonstandard $p(x)$-growth conditions," Journal of Mathematical Analysis and Applications, vol. 312, no. 1, pp. 24-32, 2005.

[33] Q. H. Zhang, "Existence of solutions for $p(x)$-Laplacian equations with singular coefficients in $\mathbb{R}^{N}$, ," Journal of Mathematical Analysis and Applications, vol. 348, no. 1, pp. 38-50, 2008.

[34] Q. Zhang, X. Liu, and Z. Qiu, "The method of subsuper solutions for weighted $p(r)$-Laplacian equation boundary value problems," Journal of Inequalities and Applications, vol. 2008, Article ID 621621, 18 pages, 2008.

[35] Q. Zhang, Z. Qiu, and X. Liu, "Existence of solutions for a class of weighted $p(t)$-Laplacian system multipoint boundary value problems," Journal of Inequalities and Applications, vol. 2008, Article ID 791762, 18 pages, 2008.

[36] R. Manásevich and J. Mawhin, "Periodic solutions for nonlinear systems with $p$-Laplacian-like operators," Journal of Differential Equations, vol. 145, no. 2, pp. 367-393, 1998. 